\title{
THE WAVE ENERGY CONVERTER CONTROL COMPETITION: OVERVIEW
}

\author{
John Ringwood* \\ Maynooth University \\ Dublin, Ireland \\ Email: john.ringwood@mu.ie
}

\author{
Francesco Ferri \\ Aalborg University \\ Aalborg, Denmark \\ Email: ff@civil.aau.dk
}

\author{
Nathan Tom \\ National Renewable Energy Laboratory \\ Golden, CO, USA \\ Email: nathan.tom@nrel.gov
}

\author{
Kelley Ruehl \\ Sandia National Laboratories \\ Albuquerque, NM, USA \\ Email:kmruehl@sandia.gov
}

\author{
Nicols Faedo \\ Maynooth University \\ Dublin, Ireland \\ Email: nicolas.faedo.2017@mumail.ie
}

\author{
Giorgio Bacelli \\ Sandia National Laboratories \\ Albuquerque, NM, USA \\ Email: gbacell@sandia.gov
}

\author{
Yi-Hsiang Yu \\ National Renewable Energy Laboratory \\ Golden, CO, USA \\ Email: yi-hsiang.yu@nrel.gov
}

\author{
Ryan G. Coe \\ Sandia National Laboratories \\ Albuquerque, NM, USA \\ Email: rcoe@sandia.gov
}

\section{ABSTRACT}

Over the past two years, a wave energy converter control systems competition (WECCCOMP) has been in progress, with the objective of comparing different wave energy converter (WEC) control paradigms on a standard benchmark problem. The target system is a point absorber, corresponding to a single float with an absolute reference, of the WaveStar WEC prototype. The system was modelled in WEC-Sim, with the hydrodynamic parameters validated against tank test data. Competitors were asked to design and implement a WEC control system for this model, with performance evaluated across six sea states. The evaluation criteria included a weighted combination of average converted power, peak/average power, and the degree to which the system physical constraints were exploited or temporarily exceeded.

This paper provides an overview of the competition, which includes a comparative evaluation of the entries and their performance on the simulation model. It is intended that this paper will act as an anchor presentation in a special session on WECCCOMP at OMAE 2019, with other papers in the special session

*Address all correspondence to this author. contributed by the competitors, describing in detail the control algorithms and the results achieved over the various sea states.

\section{KEYWORDS}

Renewable energy; wave energy converter; control system; simulation; competition

\section{INTRODUCTION}

Energy in ocean waves is distributed across a wide range of frequencies, making it a challenge to minimize the loading of a WEC while maximizing power capture across the range of sea states that a wave energy installation may be subject to. When using simple resistive damping control, even a well-designed device will fail to capture much of the energy in ocean waves [1]. As a result, a large number of studies have begun to investigate advanced control design and implementation for WECs; these studies have generally shown very attractive results for increased energy absorption, as well as performance factors such as decreased loads [1-3], and represent a key path towards lowering 
the levelized cost of energy (LCOE) for WECs [4].

While there are a significant number of studies that evaluate particular devices under particular wave excitation conditions, few studies exist (with the notable exceptions of, for example, [1] and [3]) which compare a number of control strategies on one (or a set of) standard device(s) with consistent wave excitation applied in each case, to level the playing field. However, controller evaluations are usually carried out in simulation, where the simulation model is often identical to that used to build the model-based controller. In such a situation, any controller sensitivities caused by modelling inaccuracies, such as friction and/or viscous losses, are masked in the evaluation and will be explored in the second (implementation) phase of WECCCOMP. In addition, because of the noncausal nature of the generic impedancematching control problem [5], future information (available in simulation environments) [6] is often assumed for the controller. While there are ways of estimating such future information [7], the effects of the estimation errors are not always considered [8]. In the first phase of WECCCOMP, competitors were provided up-wave measurements in the simulations that could be used to predict the excitation forces and torques at the float center of gravity. Finally, the real-time computational requirements of WEC controllers are not always clear from simulation studies (usually computed in a non-real-time environment) and will also be tested in the second phase of WECCCOMP.

Despite the fact that some comparative simulation results are available [3], there is also a desire to compare a variety of WEC control strategies under real, or at least wave tank, implementation scenarios (see, for example, [9]), so that all real effects are encountered, such as nonlinear hydrodynamic and power take off (PTO) effects; realistic measurement assumptions, including the presence of measurement noise and bias; and real-time computational requirements. In the first phase of WECCCOMP, nonlinear power take-off efficiency is included, but the remaining effects will be encountered in the second experimental phase of WECCCOMP. Ironically, the challenge for WEC controllers for small-scale WECs can be greater because of the exaggerated role of friction and the higher sampling rate requirements associated with faster dynamics, but these issues are, at least, consistent for each of the compared control strategies.

The objective of the currently proposed competition, which consists of a standard WEC prototype platform, is to compare the energy capture performance of various WEC control strategies, first in simulation and then, for shortlisted entrants, on the prototype device in a wave tank environment. In order to provide a consistent simulation environment for both competitors and evaluators, the WEC-Sim simulation environment [10] is be employed. For wave tank testing, the real-time control algorithms will be implemented using the Matlab/Simulink xPC environment.

The current status of WECCCOMP is that the simulation entries have been submitted and evaluated and the main purpose of the session containing this paper is to announce those results while exposing the details of the various approaches. The re- maining components of WECCCOMP include the implementation of each of the controllers, using a real-time system, on the experimental 1-20th scale system in the wave basin at Aalborg University, which is slated for June 2019. Following the implementation evaluation, the final experimental results will be disseminated. Some further information on WECCCOMP is available at http://www.eeng.nuim.ie/coer/wec-control-competitionreleased/.

\section{THE WEC SYSTEM}

The system to be used in the control competition is a single degree of freedom (DOF) wave-activated body WEC (Fig. 1). Though, hydrodynamically, there are multiple DOFs of the conventional coordinate system, which includes surge, heave, and pitch, these are not independent and are resolved into a single PTO DOF. The floater is connected to the fixed reference frame through a hinge (point A). At equilibrium, the floater arm stands at approx. $30^{\circ}$ with respect to the water line. The submerged volume of the floater resembles a hemisphere in the static position. The system is equipped with the following hardware:

Linear Motor and Controller - LinMot Series P0137x240F and LinMot E1200

Force Sensor - s-beam load cell, Futek LSB302 300lb, with SGA Analogue Strain Gauge Amplifier

Position Sensor - MicroEpsilon ILD-1402-600

Accelerometer - Dual-axis accelerometer, Analog Devices ADXL203EB

I/O Board - DAQ NI PCI-6221 DAQ

Additionally, real-time information about sea surface elevation at three separate points up-wave of the floater will be provided using resistive wave gauges.

The linear motor (PTO system) can be driven either as a force or position follower. For the case of the force follower, the target force can include a reactive power term. While the actuator can provide up to $\pm 200 \mathrm{~N}$, the force provided by the actuator will be constrained to the more realistic range of $\pm 60 \mathrm{~N}$. Relevant dimensions and mechanical properties of the system are listed in Table 1. Note that the linearly measured position and force will be converted into the angular motion of the WEC and the control moment, respectively, through a fully-nonlinear trigonometric calculation.

\section{Hydrodynamic Model}

The floater-wave interaction is modelled by decomposing the overall hydrodynamic force into three main (uncoupled) components.

Hydrostatic Force - Related to the buoyancy and gravity forces acting on the system.

Radiation Force - Generated by the body motion in calm water

Excitation Force - Exerted by the passing wave on a lockedin-position device. 


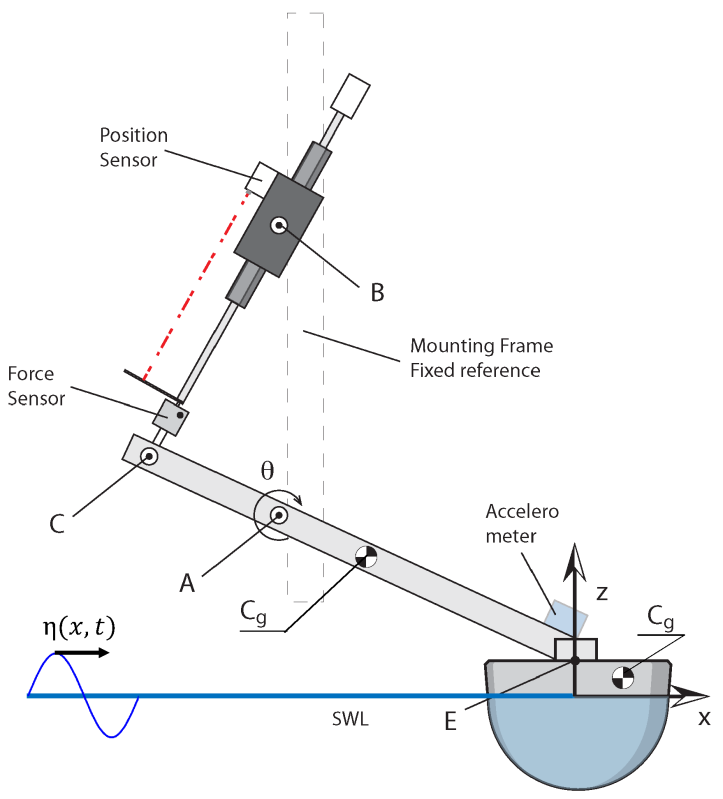

FIGURE 1. SCHEMATIC OF EXPERIMENTAL WEC SYSTEM WITH THE DIMENSIONS LISTED IN TABLE 1.

TABLE 1. WAVESTAR 1-20TH SCALE MODEL DIMENSIONS AND MASS PROPERTIES RELATIVE TO STILL WATER LINE ORIGIN.

\begin{tabular}{lr}
\hline Parameter & Value [Unit] \\
\hline Float Mass & $3.075[\mathrm{~kg}]$ \\
Float Cg (x,z) & $(0.051,0.053)[\mathrm{m}]$ \\
Float MoI (at Cg) & $0.001450\left[\mathrm{~kg} \cdot \mathrm{m}^{2}\right]$ \\
Float Draft & $0.11[\mathrm{~m}]$ \\
Float Diameter (at SWL) & $0.256[\mathrm{~m}]$ \\
Arm Mass & $1.157[\mathrm{~kg}]$ \\
Arm Cg (x,z) & $(-0.330,0.255)[\mathrm{m}]$ \\
Arm MoI (at Cg) & $0.0606\left[\mathrm{~kg} \cdot \mathrm{m}^{2}\right]$ \\
Hinge A (x,z) & $(-0.438,0.302)[\mathrm{m}]$ \\
Hinge B (x,z) & $(-0.438,0.714)[\mathrm{m}]$ \\
Hinge C (x,z) & $(-0.621,0.382)[\mathrm{m}]$ \\
\hline
\end{tabular}

The radiation force is further decomposed into a contribution related to the body velocity (radiation damping) and one proportional to the body acceleration (added mass). Similarly, the excitation force is composed of the Froude-Krylov and scattering force. These two terms are complementary as a function of the ratio of the body size to wavelength.

Both radiation and excitation force are frequency-dependent functions. For small motion, the hydrostatic force is proportional to body displacement. A linearised viscous drag term is also in-

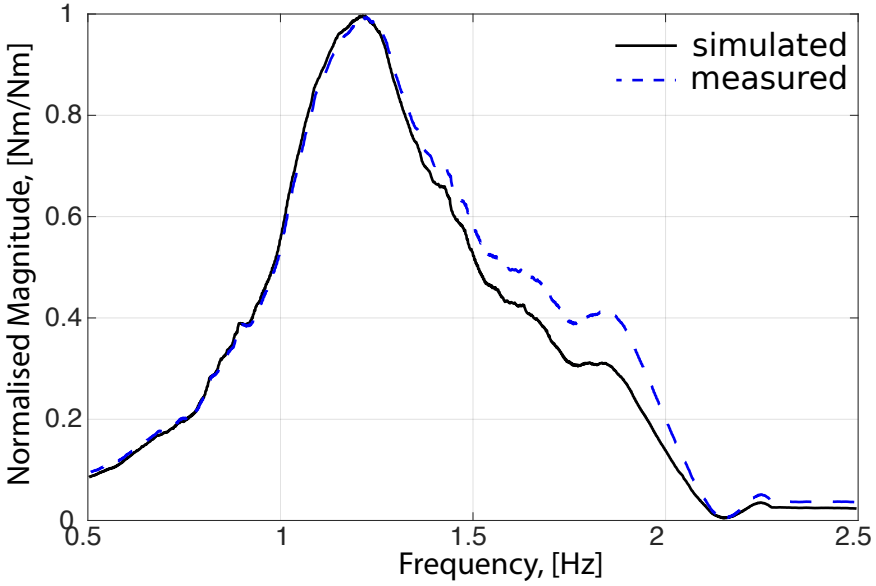

FIGURE 2. COMPARISON BETWEEN SIMULATED (BLACK) AND MEASURED (BLUE) RADIATION FORCE. THE FREQUENCY REPRESENTATION IS OBTAINED BY APPLYING THE FOURIER TRANSFORM TO A GIVEN TIME SERIES. THE MEASURED RADIATION FORCE IS OBTAINED BY SUBTRACTING THE HYDROSTATIC AND INERTIA FORCES TO THE MEASURED FORCES.

cluded in the model. Further details on the model, and its validation, can be found in [11].

\section{Hydrodynamic Parameters}

The hydrodynamic parameters of the considered WEC are obtained using the boundary element method (BEM) solver WAMIT. The coefficients are calculated at the hinge (Point A); thus, they sum the contribution from surge, heave, and pitch. The radiation and excitation force coefficients are illustrated in the frequency domain (for model validation purposes) in Fig. 2 and Fig. 3, respectively. These can be converted to time domain quantities for use in Cummins equation [12]. The calculated angular hydrostatic coefficient is $92.33 \mathrm{Nm} / \mathrm{rad}$. The radiation frequency response function has been approximated with a second order state space model, the order of which has been reduced using the Henkel singular value analysis implemented in Matlab.

\section{Model Validation}

The hydrodynamic coefficients calculated from WAMIT (Version 7.2) are compared with results obtained from experimental tests in Figs. 2, 3, and 4. Figure 2 shows the magnitude and phase plot of the Fourier transform of a radiation force time series. The measured radiation force (blue line) is calculated from the total measured moment by subtracting the hydrostatic and inertial terms. On the other hand, the calculated radiation force (black line) is obtained by filtering the measured velocity time series, for the same test, using the state space radiation model. It should be noticed that the magnitude plot is not normalised by the magnitude of the velocity signal, which is the reason for the non-smooth trend. Nevertheless, the measured and simulated radiation force show good agreement, and thus the 


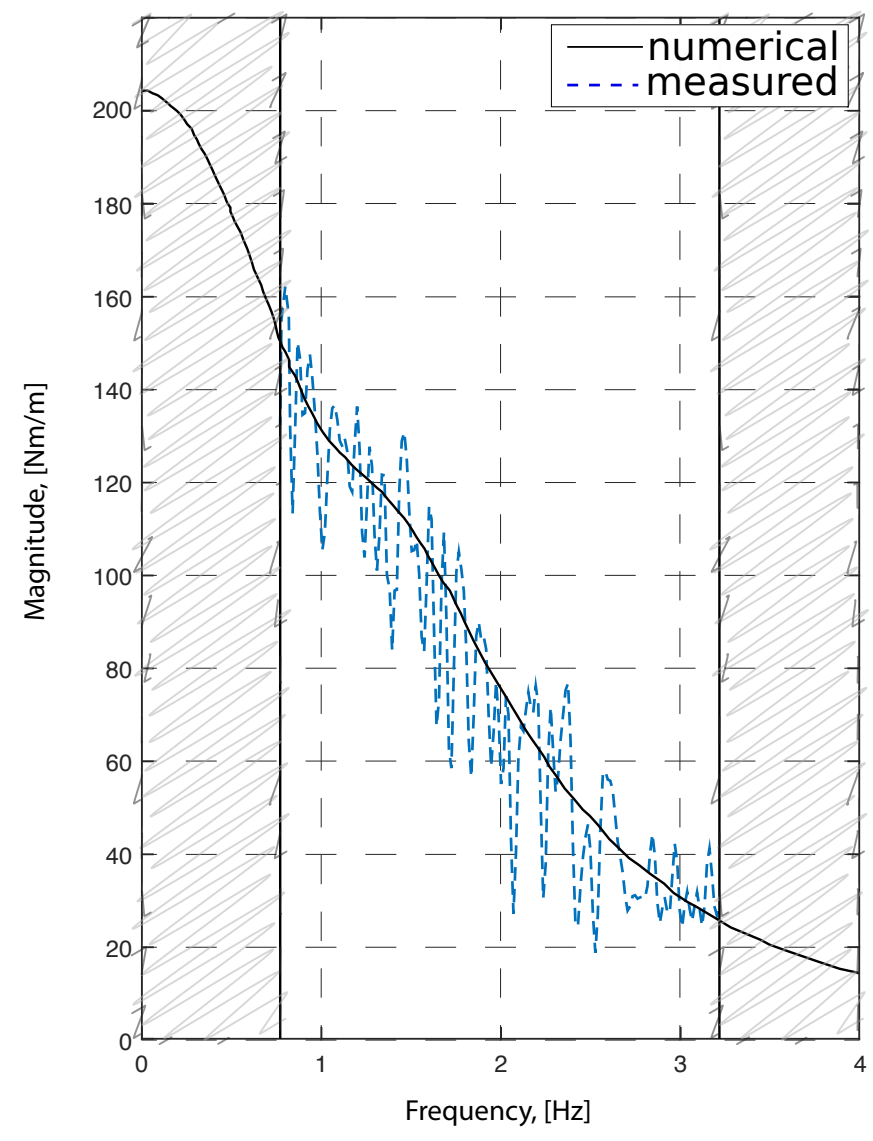

FIGURE 3. COMPARISON BETWEEN NUMERICAL (BLACK) AND MEASURED (BLUE) EXCITATION FORCE MAGNITUDE COEFFICIENTS IN FUNCTION OF THE INCOMING WAVE FREQUENCY. WITHIN THE SCRIBBLED AREAS, THE WAVE ENERGY CONTENT WAS NOT SUFFICIENT TO OBTAIN RELIABLE RESULTS (I.E., SIGNAL TO NOISE RATIO WAS NOT SUFFICIENT).

low order model of the radiation force is a valid approximation for the radiation force.

Figure 3 shows the excitation force magnitude as a function of frequency, showing both the coefficients calculated from the BEM solver (black) and the results obtained from lab tests (blue). In this case, the measurement is obtained by generating irregular waves with a floater fixed at the equilibrium position. The magnitude is then obtained by dividing the Fourier transform of the measured moment by the Fourier transform of the surface elevation.

Figure 4 shows the hydrostatic force as a function of the floater angular displacement. The red line represents the linear approximation of the nonlinear curve around the equilibrium position. To obtain the hydrostatic moment, the floater is moved to the extreme positions with a slow oscillation, removing other hydrodynamic effects. It is important to notice that all the hydrodynamic coefficients are calculated using the assumption of small motion around the equilibrium position that allow for lin-

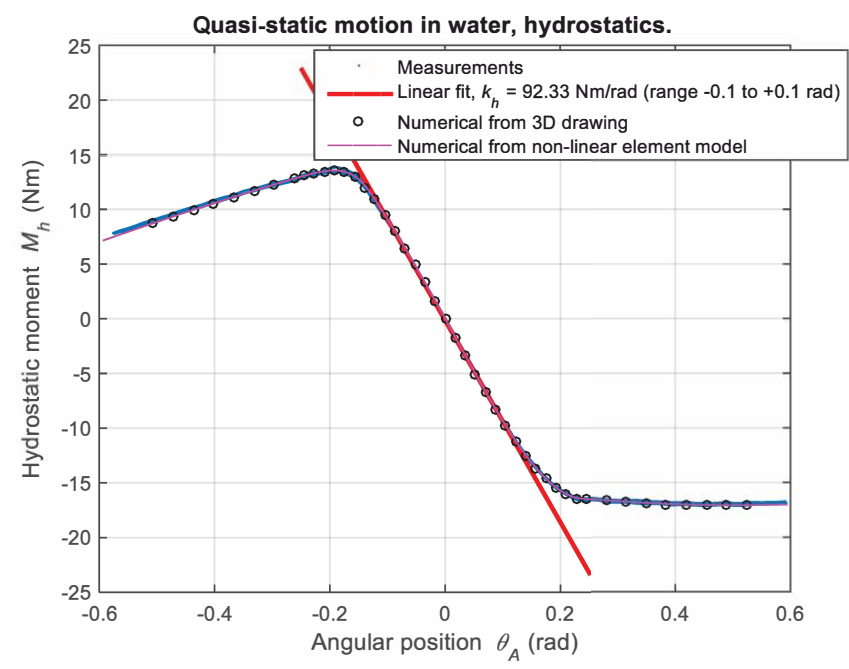

FIGURE 4. MEASURED VS NUMERICAL HYDROSTATIC MOMENT FOR THE CONSIDERED WEC.

earisation of all the force components. In Fig. 4, it is easy to see that the assumption of a linear relation between hydrostatic force and displacement is valid only in a bounded range around the equilibrium position, approx. $\pm 0.1 \mathrm{rad}$, because of how the float geometry changes with the rotation of the Wavestar arm.

\section{WEC-SIM OPEN-SOURCE CODE}

For the simulation stage of WECCCOMP, a model of the Wavestar device with control was implemented in WEC-Sim. WEC-Sim is an open-source code jointly developed by Sandia National Laboratories and the National Renewable Energy Laboratory, through funding from the U.S. Department of Energy's Water Power Technologies Office [13]. The WEC-Sim code is developed in MATLAB/Simulink, and uses Simscape Multibody to solve for a WEC's rigid body dynamics. WEC-Sim's implementation is a collection of MATLAB scripts (*.m files) and Simulink libraries (*.sl $x$ files), which are hosted on an opensource GitHub repository [14], with the current v3.1 version released in December 2018.

WEC-Sim is a time-domain code that solves for the system dynamics of WECs consisting of a combination of rigid bodies, PTO systems, mooring systems, and control systems. The dynamic response in WEC-Sim is calculated by solving the WEC's equation of motion for each rigid body about its center of gravity, in 6 DOFs, based on Cummins' formulation [12].

The WEC-Sim source code includes a preprocessing BEM input/output code that imports hydrodynamic data generated by the potential flow solvers WAMIT, NEMOH, or AQWA, and parses the BEM data into a $(* . h 5)$ data structure that is read by WEC-Sim. For more information about WEC-Sim theory, implementation, functionality, and application, refer to the WECSim website [13]. 


\section{WEC-SIM NUMERICAL MODEL}

A WEC-Sim model of the Wavestar device was described in [11] to accurately represent the physical Wavestar model that will be tested during the experimental stage of WECCCOMP. The numerical model includes the float's hydrodynamic response as well as the physical inertia of linkages and bearings. The WEC-Sim Simulink model includes the float as a hydrodynamic body block. The connection between the float and arm is a fixed connection. Similarly, revolute joints A, B, and C are modeled by revolute constraints in WEC-Sim. The WEC's nonhydrodynamic bodies consist of the following: arm, frame, Rod $\mathrm{BC}$, and motor linear actuator mass. The movement of Rod BC is modeled by a translational PTO (linear motor), which is actuated based on the control algorithm written in the competitor's controller block. The WECCCOMP controller may use inputs from the upstream wave gauge(s) and either the linear force and displacement of the motor, or the rotary torque and displacement of the float. The numerical model of the Wavestar device was provided to WECCCOMP contestants for development of their controller through a GitHub repository [15]. In addition, the hydrodynamic BEM solution obtained from WAMIT [16] was provided to the contestants to limit discrepancies between competitor numerical models. Details on the validation of the numerical model against experimental results from wave tank tests are provided in [11].

\section{EVALUATION CRITERIA FUNCTION}

The initial evaluation of controllers was composed of the following components as described in [17]:

\section{Average Extracted Power}

2. Capacity Factor - Peak power (95\% percentile) over RMS.

3. Peak PTO Force - The $95 \%$ percentile of PTO force

4. PTO Utilisation Factor - Ratio of peak PTO force and RMS PTO force

Based on further consideration, the evaluation metric was updated from its original format in [17]. The WECCCOMP simulation submissions were evaluated and compared against one another using the following evaluation criterion (EC):

$$
E C=\frac{\operatorname{avg}(P)}{2+\frac{|f|_{98}}{F_{\max }}+\frac{|z|_{98}}{Z_{\max }}+\frac{\operatorname{avg}|P|}{|P|_{98}}}
$$

where $\operatorname{avg}(P)$ is the average electrical power output from a PTO with a mechanical-to-electrical efficiency of $70 \%$ (in $\mathrm{W}$ ), $|f|_{98}$ is the $98^{\text {th }}$ percentile of the absolute motor force time history (in $\mathrm{N}), F_{\max }$ is the motor force constraint on the PTO $(60 \mathrm{~N}),|z|_{98}$ is the $98^{\text {th }}$ percentile of the absolute motor displacement time history (in $\mathrm{m}$ ), $Z_{\max }$ is the motor displacement constraint on the PTO $(0.08 \mathrm{~m}), \operatorname{avg}|P|$ is the mean absolute electrical power (in $\mathrm{W})$, and $|P|_{98}$ is the $98^{\text {th }}$ percentile of the absolute power time history (in W).
TABLE 2. EVALUATION SEA STATES.

\begin{tabular}{ccccc}
\hline Sea State & $H_{S}[\mathrm{~m}]$ & $T_{p}[\mathrm{~s}]$ & $\gamma$ & $P_{w}[\mathrm{~W} / \mathrm{m}]$ \\
\hline SS1 & 0.0208 & 0.988 & 1 & 0.182 \\
SS2 & 0.0625 & 1.412 & 1 & 2.556 \\
SS3 & 0.1042 & 1.836 & 1 & 9.390 \\
SS4 & 0.0208 & 0.988 & 3.3 & 0.192 \\
SS5 & 0.0625 & 1.412 & 3.3 & 2.735 \\
SS6 & 0.1042 & 1.836 & 3.3 & 10.00 \\
\hline
\end{tabular}

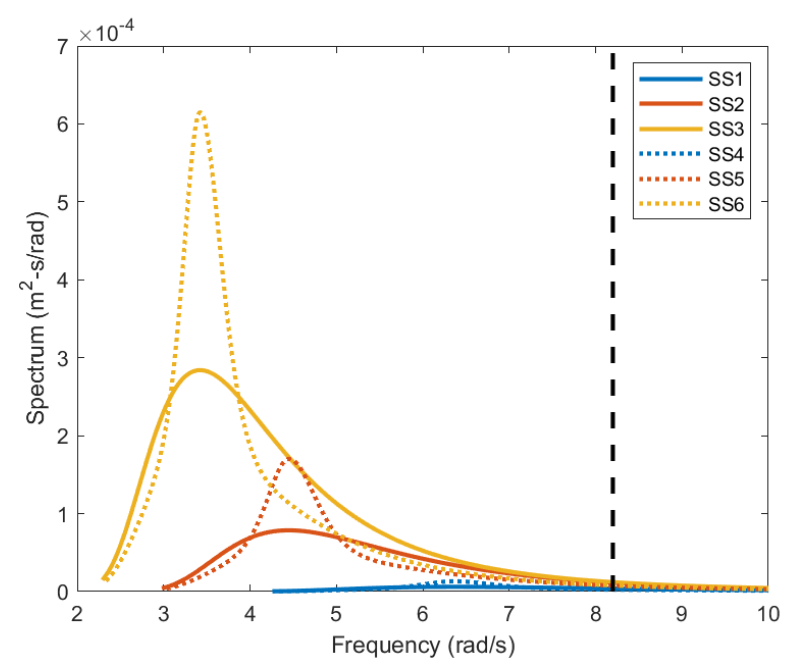

FIGURE 5. WAVE SPECTRA FOR THE SEA STATES USED IN CONTROLLER EVALUATION, AND FLOAT HEAVE RESONANCE.

The goal of the control submissions will be to maximize the EC, which acts as a benefit-to-cost ratio. While Eqn. (1) does not represent a direct measure of LCOE, it is an attempt to approximate related LCOE impacts at a very high level. Note that the physical constraints, $F_{\max }$ and $Z_{\max }$, are not rigidly enforced in the simulation evaluation. In fact, $F_{\max }$ and $Z_{\max }$ can be exceeded in simulation. Rather, their relativity to $|f|_{98} \mid$ and $|z|_{98}$ (respectively) are captured in the EC. This approach was chosen for the simulation to avoid a situation in which both of the constraints cannot be simultaneously satisfied. However, strict control force limits will be used in the experimental evaluation.

A total of six sea states were selected to evaluate the controllers (see Table 2). These sea states were selected based on their energy content relative to the response of the scale WaveStar WEC. The wave spectra of the sea states are plotted along with the heave natural resonance in Fig. 5. Note the disparity between the sea spectral peaks and the natural device resonant frequency. This was chosen deliberately in order to exercise the controllers. 


\section{SUMMARY OF CONTROL APPROACHES}

Three completed entries were submitted and, unsurprisingly, most focus on model predictive control (MPC)-like algorithms [18], probably reflecting the need to be conscious of adhering to the physical system constraints, as they are explicitly articulated in the competition performance criteria. However, there is some variation in both the type of model used for the controller optimisation and the algorithms employed for wave excitation estimation. For the control model, one method utilised a standard statespace model, while another used a trapezoidal discretization and the third entry utilised a Gaussian process (data-based) model. Not all competitors used the available wave measurements, and forecasting algorithms vary from Guassian process-based methods to autoregressive (with exogenous input) models and Kalman filter-based philosophies.

\section{Competitor 1}

The control algorithm is essentially based around an MPC formulation, with a time step of $50 \mathrm{~ms}$. The wave excitation force is estimated using a Kalman filter and an extended Kalman filter is used to solve the nonlinear multi-step ahead excitation force prediction problem, with a time step of $50 \mathrm{~ms}$. One interesting variation in the MPC algorithm used is that the weightings used in the quadratic program are optimised offline using extensive simulation and then a sea state recognition algorithm is employed online to select the correct tuning. This recognition algorithm employs an unscented Kalman filter. The MPC algorithm used is based on a proprietary algorithm that uses trapezoidal discretization of the performance function and has been patented.

\section{Competitor 2}

This entry also uses an MPC controller, which has been previously published in the literature [19]. The performance function is proportional to absorbed power, with a penalty term on PTO force. The quadratic problem is solved using an active set optimisation strategy. A Kalman filter uses a disturbanceaugmented model of the system to estimate both the states of the system and the wave excitation force, while an autoregressive with exogenous input (ARX) model is used for excitation force forecasting, using up-wave wave elevation as the exogenous input. The controller parameters are not adapted with sea state, but are static, with any adjustment with sea state being handled by the excitation force prediction.

\section{Competitor 3}

The controller employed by this group could be considered to be an MPC type, but the model employed, a Gaussian process (GP) model, is not commonly used by MPC algorithms, and is somewhat unique in the wave energy application space. The complete algorithm employs a model-based wave excitation force estimator, which is independent of the up-wave (or other) wave gauge measurements available. An artificial neural network is used to deduce the free surface elevation, from the estimate of the wave excitation force. The excitation force prediction is performed with a GP model and the control is GP-based, which is data- rather than model-based and can give good information about uncertainty in the prediction. Adaptation across sea states is performed by tuning parameters associated with the GP-based functions.

\section{SUMMARY OF SIMULATION RESULTS}

For each sea state, the WECCCOMP competitors were instructed to simulate their controller for a duration of $100 T_{p}$ with a ramp time of $5 T_{p}$. The first 25 seconds of the WEC-Sim simulation were discarded, allowing the start-up transients to disappear before calculating competition metrics. The WECCCOMP organizers reran all competitor submissions, compared the simulation results to submitted time series and recalculated the EC for each of the six sea states. This was completed to ensure the competitors' results were reproducible and could be used in other test cases, as required.

During this process it was found that, based on the submitted results, Competitor 3 only ran simulations for $50 T_{p}$, while Competitor 1 and Competitor 2 both submitted the required $100 T_{p}$ duration time histories. Furthermore, when simulating Competitor 3's controller in SS4 for the required $100 T_{p}$ duration, the simulation did not proceed to completion and crashed $2.52 \mathrm{~s}$ before the end-time. For this sea state, the WECCCOMP organizers decided to use only the available time history when calculating the competition metrics. The loss of $2.52 \mathrm{~s}\left(\approx 2.5 T_{p}\right)$ of simulation time amounted to just over $3 \%$ of the total evaluation simulation time and the organizers decided this would have minimal impact on the EC calculation for SS4. The organizers also felt that it would have been unfair to notify Competitor 3 of the controller error and request an updated submission as this would have provided additional time to tune the controller not provided to the other competitors.

\section{Comparison of Competition Metrics}

As observed from Eqn. (1), there are four terms that must be calculated from the post-processed simulation time histories. The electrical time averaged power output (ETAP), calculated for each sea state and each competitor, is plotted in Fig. 6. For all sea states, except SS1, Competitor 1's controller produced the greatest ETAP. Competitor 2 is a close second, coming within 5\% of Competitor 1 on average, while Competitor 3 at best comes within 10\% of Competitor 1 with major reductions in SS1, a $84 \%$ reduction, and SS4, a $37 \%$ reduction.

The 98th percentiles for the linear motor force and displacement are plotted in Figs. 7 and 8 respectively. As observed from EC (Eqn. (1)), the motor force and displacement metrics are positive and lead to an increase in the denominator, potentially decreasing EC. Therefore, the competitors' controllers should attempt to minimize these quantities to improve the sea state EC score. For the linear motor force, Competitor 3's controller gen- 


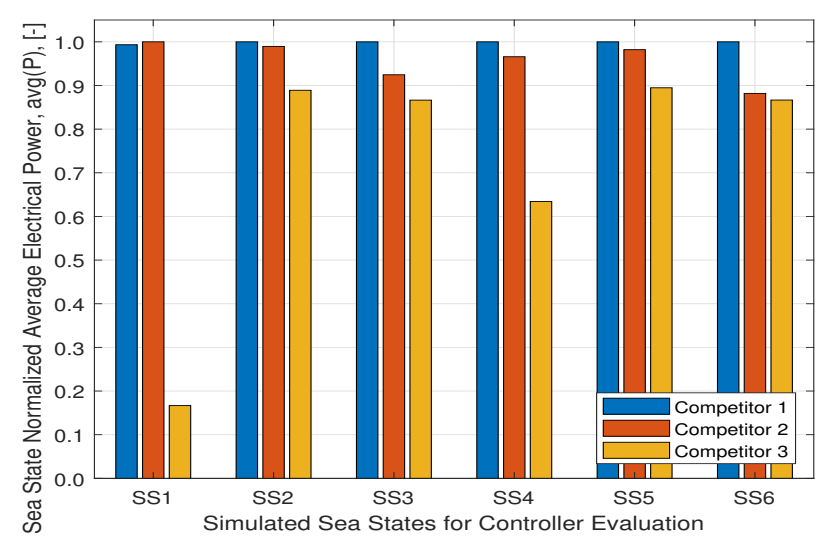

FIGURE 6. COMPETITOR $\operatorname{avg}(P)$ FOR EACH SEA STATE. THE RESULTS HAVE BEEN NORMALIZED BY THE GREATEST VALUE FROM THE COMPETITORS FOR EACH SEA STATE.

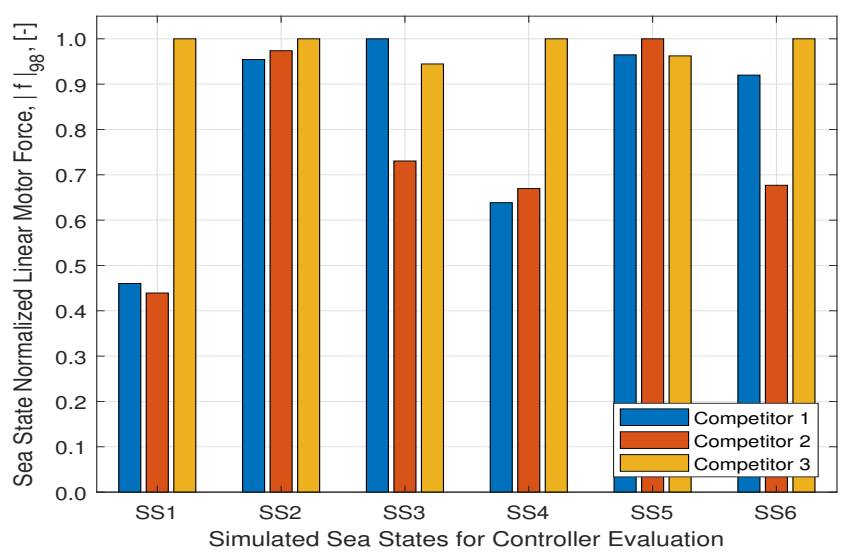

FIGURE 7. COMPETITOR $|f|_{98}$ FOR EACH SEA STATE. THE RESULTS HAVE BEEN NORMALIZED BY THE GREATEST VALUE AMONG THE COMPETITORS FOR EACH SEA STATE.

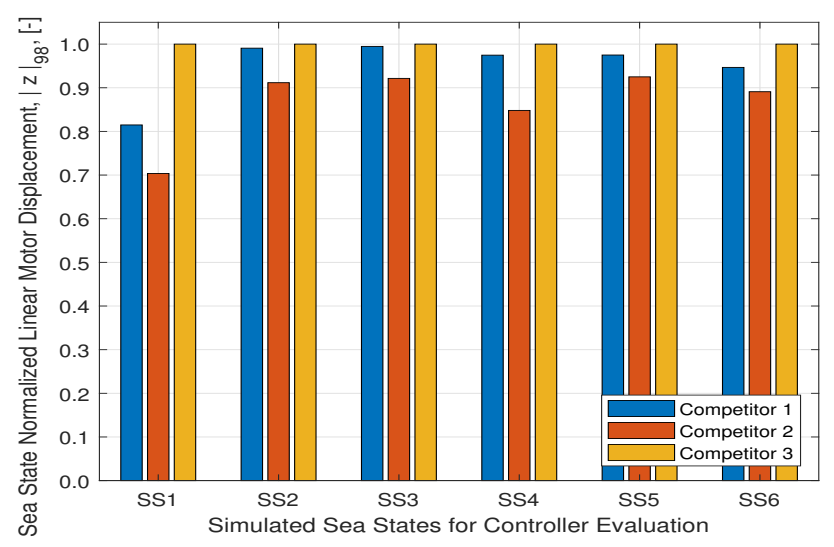

FIGURE 8. COMPETITOR $|z|_{98}$ FOR EACH SEA STATE. THE RESULTS HAVE BEEN NORMALIZED BY THE GREATEST VALUE AMONG THE COMPETITORS FOR EACH SEA STATE. $\cdot / /$ www nverleaf com/7514853787kmwermdnztkd

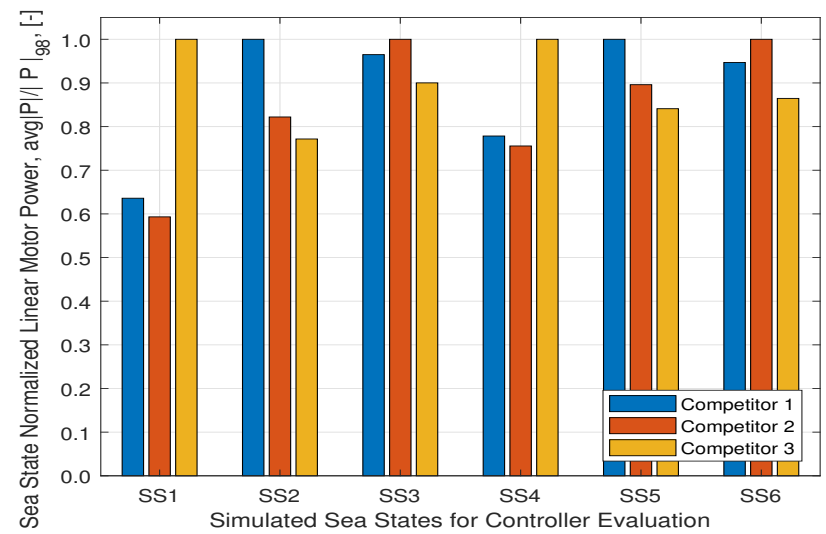

FIGURE 9. COMPETITOR $a v g|P| /|P|_{98}$ FOR EACH SEA STATE. THE RESULTS HAVE BEEN NORMALIZED BY THE GREATEST VALUE AMONG THE COMPETITORS FOR EACH SEA STATE.

erally produced the highest values, most notably in SS1 and SS4. The exceptions are SS3 and SS5, where the controllers developed by Competitors 1 and 2 produce the greatest values respectively. Following the linear motor force, Competitor 3's controller also produces the largest motor displacement for every sea state, followed by Competitor 1, and then by Competitor 2 .

The 98th percentile for the ratio of the instantaneous electrical to the average absolute value of electrical motor power is plotted in Fig. 9. The avg $|P| /|P|_{98}$ metric has a negative contribution to the denominator of Eqn. (1), which decreases the denominator value and potentially increases EC. In order to improve the EC, the developed controllers should attempt to maximize this quantity, which has a maximum value of 1 . The results are mixed, as each competitor produced the greatest values in two sea states; however, the sea states do share the same peak period. Competitor 3 produced the greatest metric values in SS1 and SS4 by more than $25 \%$, but it was not enough to offset the decrease in the ETAP; refer to Fig. 6. Competitor 1 produced the largest values in SS2 and SS5, while Competitor 2 produced the largest values in SS3 and SS6.

The competitor EC score, for each sea state, is plotted in Fig. 10 and the average EC score across all sea states is shown in Table 3. The largest contribution to the average EC score comes from SS3 and SS6, which is to be expected, given the increased energy content in the sea state (Fig. 5); however, what is interesting is that, despite the reduced spread of energy in SS6, the EC score remains close to SS3. After calculating the final mean EC score, Competitor 1 has come in first place, Competitor 2 comes in a very close second, and Competitor 3 comes in third with a more significant reduction in EC, see Table 3.

\section{Comparison of Simulation Time Histories}

The EC and the performance metrics provide a statistical representation to compare controller performance, but a comparison of the time and frequency domain results remains important. The time and frequency domain comparisons of the linear 


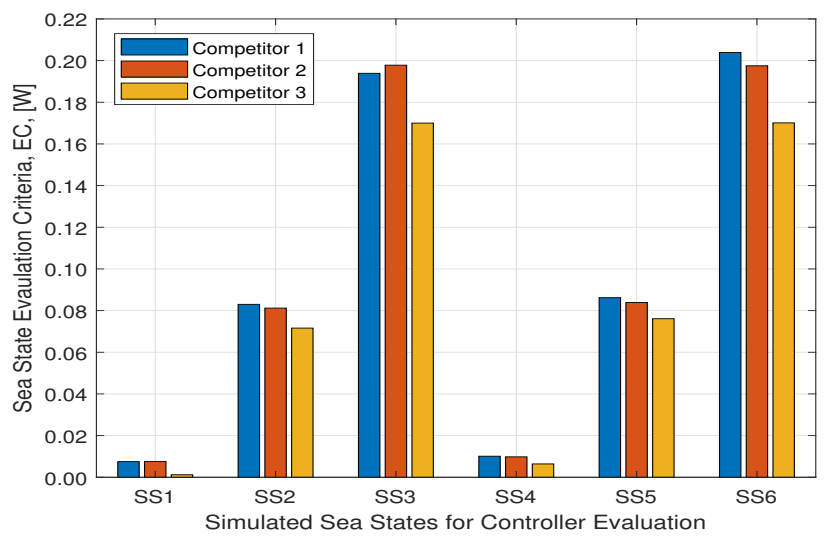

FIGURE 10. COMPETITOR EC FOR EACH SEA STATE.

motor force are presented in Fig. 11 for SS6. The time series in Fig. 11(a) shows that, for each controller, the commanded force from the motor are all in phase, with the main variation coming from the minimum and maximum values of the commanded force. The time history from Competitors 1 and 2 match very closely until Competitor 2 reaches a self-imposed ceiling of approximately $0.7 F_{\text {max }}$, while Competitor 1 runs up to $F_{\text {max }}$. Competitor 3 , in several instances, exceeds $F_{\max }$ when commanding a positive motor force, but matches Competitor 1 more closely when commanding a negative motor force.

An interesting result is found in Fig. 11(b), which sorts the absolute value of the force time history in ascending order, and marks the 98th percentile used in the EC calculation. This plot shows that, up to the 91 st percentile, Competitor 3 has the lowest commanded force values; however, past this point, the growth in peak force increases almost exponentially, while the commanded torque from Competitors 1 and 2 are curtailed and produce flatter curves. This is supported by Fig. 11(c), which plots the frequency response of the commanded motor force. This FFT analysis shows Competitor 1 has the largest force magnitudes, leading to the elevated force values at lower percentiles in Fig. 11(b). The phase of the commanded force then accounts for Competitor 1 having a lower $|f|_{98}$ compared to Competitor 3 . Therefore, the few instances where Competitor 3 exceeded $F_{\max }$ has driven its 98th percentile significantly higher than the other competitors, leading to the drop in EC for SS6.

As any WEC must generate power, it is of interest to examine the motor electrical output that would be sent to the grid. Time and frequency domain comparisons of the linear motor electrical power, for SS6, can be found in Fig. 12. Similarly to the commanded motor force, the generated electrical power time histories, Fig. 12(a), have very similar phasing, with the largest swings in bidirectional power flow given by Competitor 3. This is further illustrated in Fig. 12(b) where, past the 95th percentile, the peaks in electrical power for Competitor 3 increase at a faster rate than for Competitors 1 or 2 . However, the sorted avg|P|/|P| curves are more closely packed together when compared against the commanded force in Fig. 11(b). The fast Fourier transform analysis of the linear motor electrical power, shown in Fig.12(c),

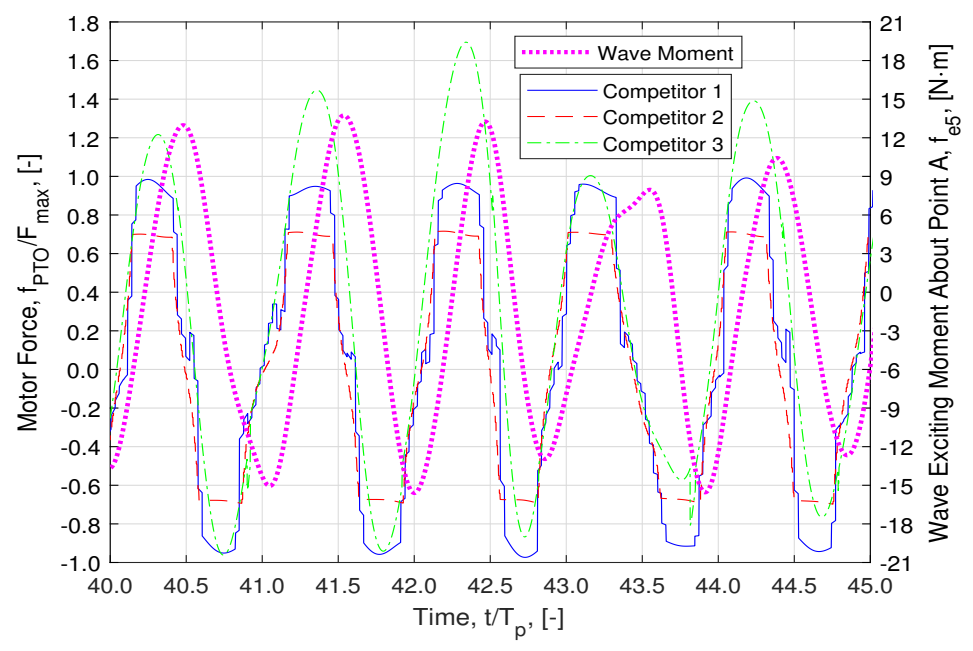

(a) TIME SERIES OF WAVESTAR MOTOR FORCE AND WAVE MOMENT

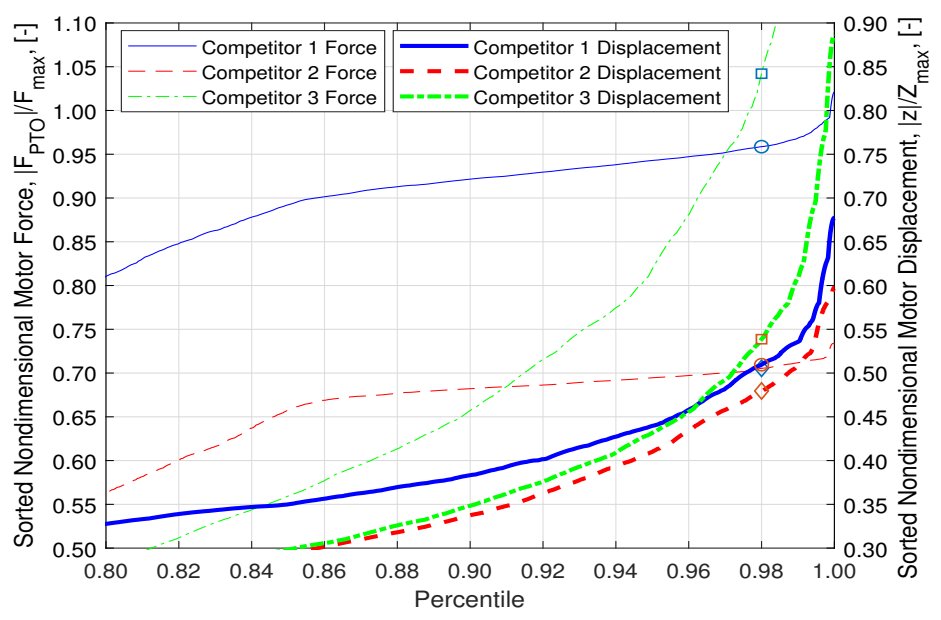

(b) SORTED WAVESTAR MOTOR FORCE AND DISPLACEMENT

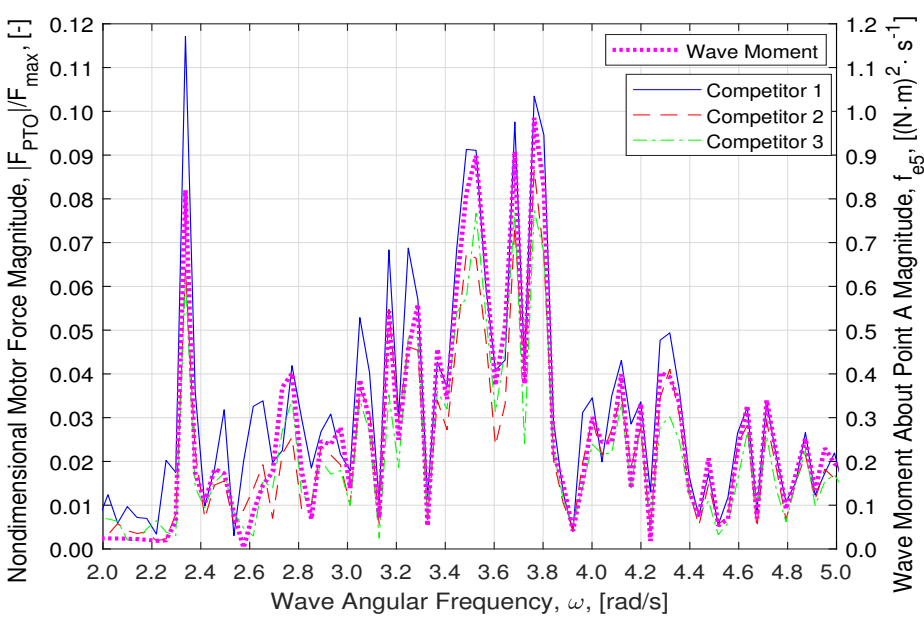

(c) WAVESTAR MOTOR FORCE AND WAVE FREQUENCY RESPONSE

FIGURE 11. TIME AND FREQUENCY DOMAIN COMPARISON OF THE MOTOR FORCE FROM EACH COMPETITORS CONTROLLER IN SEA STATE 6. 
TABLE 3. EVALUATION CRITERIA AVERAGED OVER ALL SEA STATES.

\begin{tabular}{|c|c|c|c|}
\hline EC & Competitor 1 & Competitor 2 & Competitor 3 \\
\hline Averaged & 0.0975 & 0.0963 & 0.0826 \\
\hline Normalized & 1 & 0.9878 & 0.8471 \\
\hline
\end{tabular}

shows significant variation in frequency content between competitors. At the lower electrical frequencies, because power will have approximately two oscillations per one wave oscillation, Competitor 3 has the largest magnitude in consumed and generated power; however, after passing $7.5 \mathrm{rad} / \mathrm{s}$, the magnitude of the frequency response for Competitor 2 is the greatest.

\section{CONCLUSIONS}

The competition has stimulated thinking about how to approach the design of deployable WEC controllers (for the competitors), because no unavailable information, such as future wave elevation, is available and the system contains nonideal components, such as nonideal PTO efficiency, physical PTO limits, measurement noise, etc. However, the first stage of the competition, involving a simulation evaluation, relaxes some of these aspects (e.g., no physical limits, absence of noise, etc), which will be fully present in the experimental evaluation. The competition has forced the organisers to carefully consider a realistic evaluation criterion, which is predominantly based on power production, but also reflective of other factors that impact economic performance, such as peak-to-average ratios, etc.

By and large, the results achieved by all competitors score close to one another with respect to the evaluation criterion, with some disparities for particular sea states. This possibly reflects the general similarity of the control approaches employed, which is encouraging, as some level of consensus may finally be emerging as to the most promising direction for WEC control design. All of the control designs directly address issues such as physical PTO limits, non-ideal PTO efficiency, and excitation force forecasting. One of the main assets of the competition, we believe, is the comparison of each controller on a diverse set of sea states, but common to each controller. It is one of the rare times that such an evaluation has been carried out.

We look forward to the next stage of the competition, in which each set of competitors will be assisted in implementing their control systems on the experimental rig, slated for early March 2019. It will be interesting to see what consistency the experimental results bear to the simulation results; any disparities may reflect as much on the accuracy of the simulation model as on the potential of the individual controllers!

\section{ACKNOWLEDGEMENTS}

The Alliance for Sustainable Energy, LLC (Alliance) is the manager and operator of the National Renewable Energy Laboratory. NREL is a national laboratory of the U.S. Department of Energy, Office of Energy Efficiency and Renewable Energy.

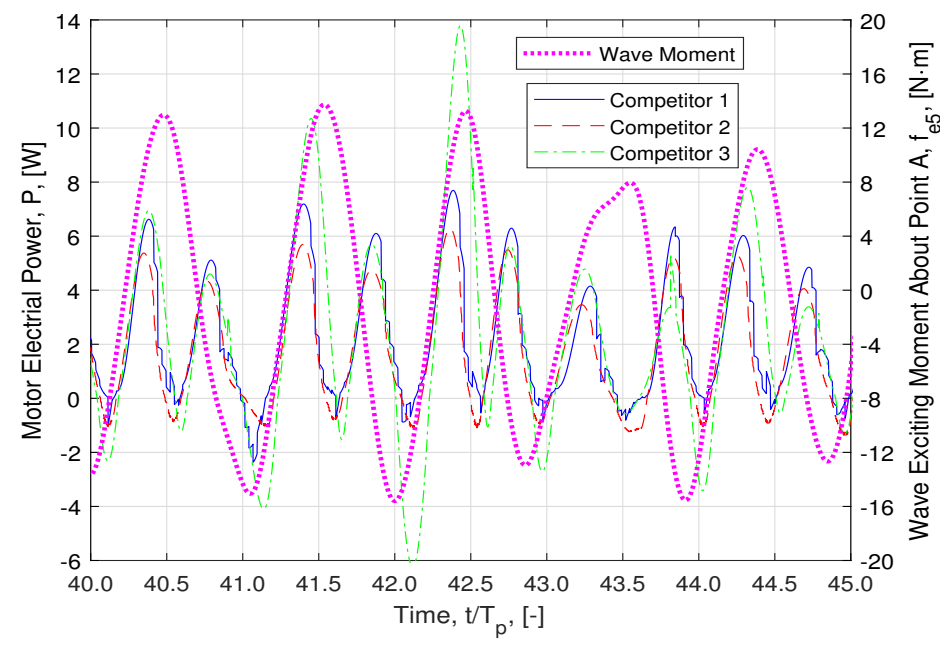

(a) TIME SERIES OF WAVESTAR MOTOR POWER AND WAVE MOMENT

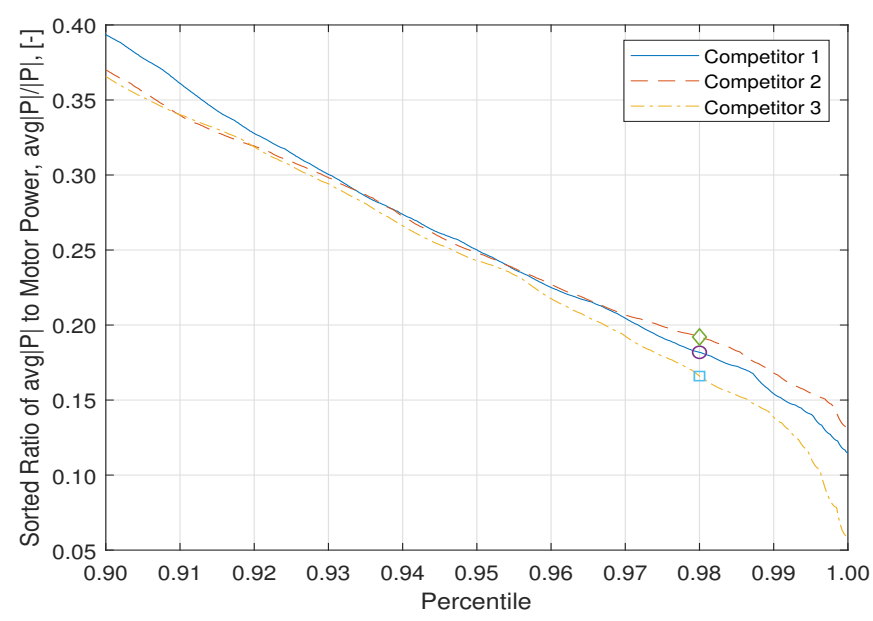

(b) SORTED RATIO OF $a v g|P|$ TO WAVESTAR MOTOR POWER

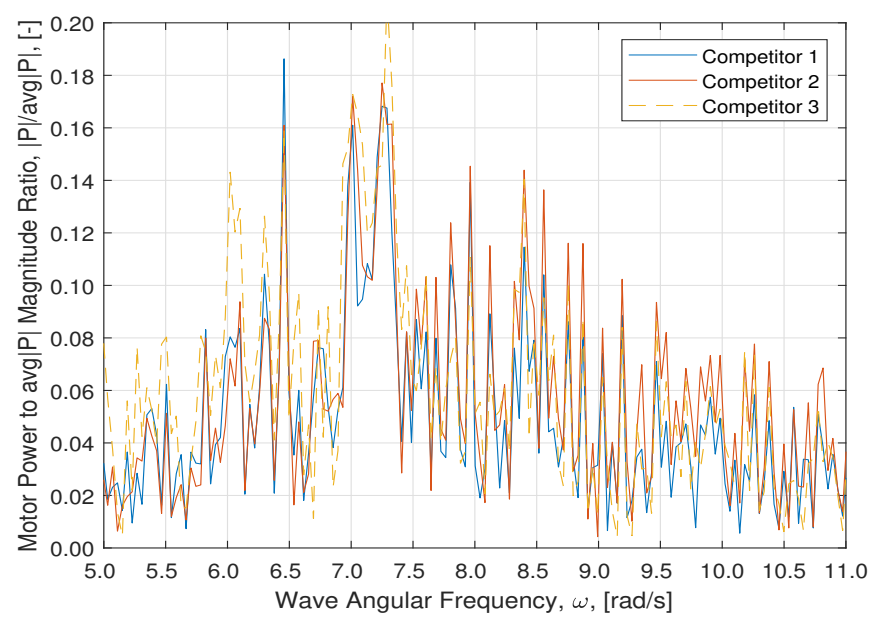

(c) WAVESTAR MOTOR FORCE AND WAVE FREQUENCY RESPONSE

FIGURE 12. TIME AND FREQUENCY DOMAIN COMPARISON OF THE MOTOR ELECTRICAL POWER FROM EACH COMPETITOR'S CONTROLLER IN SEA STATE 6. 
This work was authored by the Alliance and supported by the U. S. Department of Energy under Contract No. DE-AC3608GO28308. Sandia National Laboratories is a multimission laboratory managed and operated by National Technology and Engineering Solutions of Sandia, LLC., a wholly owned subsidiary of Honeywell International, Inc., for the U.S. Department of Energys National Nuclear Security Administration under contract DE-NA0003525. Funding was provided by the U.S. Department of Energy Water Power Technologies Office. The views expressed in the article do not necessarily represent the views of the U.S. Department of Energy or the U.S. government. The U.S. government retains, and the publisher, by accepting the article for publication, acknowledges that the U.S. government retains a nonexclusive, paid-up, irrevocable, worldwide license to publish or reproduce the published form of this work, or allow others to do so, for U.S. government purposes.

J. Ringwood and N. Faedo are grateful for the financial support of Science Foundation Ireland under Grants 13/IA/1886 and 12/RC/2302.

\section{REFERENCES}

[1] Hals, J., Falnes, J., and Moan, T., 2011. "A comparison of selected strategies for adaptive control of wave energy converters," Journal of Offshore Mechanics and Arctic Engineering, 133(3), March, p. 031101.

[2] Ferri, F., Ambühl, S., Fischer, B., and Kofoed, J. P., 2014. "Balancing power output and structural fatigue of wave energy converters by means of control strategies," Energies, 7(4), pp. 2246-2273.

[3] Wilson, D., Bacelli, G., Coe, R. G., Bull, D. L., Abdelkhalik, O., Korde, U. A., and Robinett III, R. D., 2016. A comparison of WEC control strategies, Sandia National Labs, Albuquerque, New Mexico, April 2016.

[4] Neary, V. S., Lawson, M., Previsic, M., Copping, A., Hallett, K. C., Labonte, A., Rieks, J., Murray, D., et al., 2014. Methodology for design and economic analysis of marine energy conversion (mec) technologies.

[5] Ringwood, J. V., Bacelli, G., and Fusco, F., 2014. "Energymaximizing control of wave-energy converters: The development of control system technology to optimize their operation," IEEE Control Systems, 34(5), pp. 30-55.

[6] Fusco, F., and Ringwood, J. V., 2012. "A study of the prediction requirements in real-time control of wave energy converters," IEEE Transactions on Sustainable Energy, 3(1), pp. 176-184.

[7] Fusco, F., and Ringwood, J. V., 2010. "Short-term wave forecasting for real-time control of wave energy converters," IEEE Transactions on sustainable energy, 1(2), pp. 99-106.

[8] Fusco, F., and Ringwood, J. V., 2011. "A model for the sensitivity of non-causal control of wave energy converters to wave excitation force prediction errors," In Proceedings of the 9th European Wave and Tidal Energy Conference (EWTEC), School of Civil Engineering and the Environment, University of Southampton.

[9] Nguyen, H.-N., Sabiron, G., Tona, P., Kramer, M. M., and Sanchez, E. V., 2016. "Experimental validation of a nonlinear mpc strategy for a wave energy converter prototype," In ASME 2016 35th International Conference on Ocean, Offshore and Arctic Engineering, American Society of Mechanical Engineers, pp. V006T09A019-V006T09A019.

[10] Ruehl, K., Yu, Y.-H., M., L., and Michelen, C., 2014. "Preliminary verification and validation of wec-sim, an opensource wave energy converter design tool," In Proc. of the ASME 33rd International Conference on Ocean, Offshore and Arctic Engineering (OMAE).

[11] Tom, N., Ruehl, K., and Ferri, F., 2018. "Numerical Model Development and Validation for the WECCCOMP Control Competition,' In Proceedings of the 38th International Conference on Ocean, Offshore and Arctic Engineering (OMAE), Madrid, Spain.

[12] Cummins, W., 1962. The Impulse Response Function and Ship Motions, David Taylor Model Dasin (DTNSRDC).

[13] WEC-Sim - Wave Energy Converter SIMulator. http://wecsim.github.io/WEC-Sim/. Accessed: 1-2018.

[14] WEC-Sim GitHub Repository v3.0. https://github.com/WEC-Sim/WEC-Sim.

[15] WECCCOMP GitHub Repository. https://github.com/WEC-Sim/WECCCOMP. Accessed: 12-15-2018.

[16] Lee, C., and Newman, J., 2016. WAMIT User Manual Version 7.2.

[17] Ringwood, J., Ferri, F., Ruehl, K. M., Yu, Y.-H., Coe, R., Bacelli, G., Weber, J., and Kramer, M., 2017. "A competition for WEC control systems," In Proceedings of the 12th European Wave and Tidal Conference (EWTEC), Cork, Ireland.

[18] Faedo, N., Olaya, S., and Ringwood, J., 2017. "Optimal control, mpc and mpc-like algorithms for wave energy systems: An overview," submitted IFAC Journal of Systems and Control.

[19] Richter, M., Magaña, M. E., Sawodny, O., and Brekken, T. K., 2014. "Power optimisation of a point absorber wave energy converter by means of linear model predictive control," IET Renewable Power Generation, 8(2), pp. 203215. 